\title{
A Simple Opto-Fluidic Switch Detecting Liquid Filling in Polymer-Based Microfluidic Systems
}

Bundgaard, Frederik; Geschke, Oliver; Zengerle, R; Ducree, J

Published in:

International Solid-State Sensors, Actuators and Microsystems Conference, 2007. TRANSDUCERS 2007.

Link to article, DOI:

10.1109/SENSOR.2007.4300241

Publication date:

2007

Document Version

Publisher's PDF, also known as Version of record

Link back to DTU Orbit

Citation (APA):

Bundgaard, F., Geschke, O., Zengerle, R., \& Ducree, J. (2007). A Simple Opto-Fluidic Switch Detecting Liquid Filling in Polymer-Based Microfluidic Systems. In International Solid-State Sensors, Actuators and Microsystems Conference, 2007. TRANSDUCERS 2007. IEEE. https://doi.org/10.1109/SENSOR.2007.4300241

\section{General rights}

Copyright and moral rights for the publications made accessible in the public portal are retained by the authors and/or other copyright owners and it is a condition of accessing publications that users recognise and abide by the legal requirements associated with these rights.

- Users may download and print one copy of any publication from the public portal for the purpose of private study or research.

- You may not further distribute the material or use it for any profit-making activity or commercial gain

- You may freely distribute the URL identifying the publication in the public portal 


\title{
A SIMPLE OPTO-FLUIDIC SWITCH DETECTING LIQUID FILLING IN POLYMER-BASED MICROFLUIDIC SYSTEMS
}

\author{
F. Bundgaard ${ }^{1}$, O. Geschke ${ }^{1}$, R. Zengerle ${ }^{2,3}$, J. Ducrée Zu $^{2,3}$ \\ ${ }^{1} \mathrm{MIC}$ - Dept. of Micro and Nanotechnology, Technical University of Denmark \\ Kgs. Lyngby, DENMARK \\ (Tel: +45 4525 6301; E-mail: og@mic.dtu.dk) \\ ${ }^{2}$ IMTEK - University of Freiburg, Laboratory for MEMS Applications \\ Freiburg, GERMANY \\ (Tel: +49 761203 7459; E-mail: jens.ducree@hsg-imit.de) \\ ${ }^{3}$ HSG-IMIT \\ Villingen-Schwenningen, GERMANY
}

\begin{abstract}
A novel detection scheme for detection of liquid levels and bubbles in microfluidic systems, using the principle of total internal reflection (TIR) is presented. A laser beam impinges on the side walls of a channel which are inclined at $45^{\circ}$. In an unfilled channel of such a "V-groove", TIR deflects the beam by $90^{\circ}$ into a simple light detector. Upon the presence of liquid, the refractive index in the channel changes, thus eliminating deflection by TIR. The detection principle is robust, requiring no calibration, and tolerating alignment errors of the laser larger than the width and depth of the microfluidic channels. The machining of the V-groves can seamlessly be integrated into common polymer microfabrication schemes such as injection molding.
\end{abstract}

Keywords: Polymer microsystems, total internal reflection, liquid level detection, gas bubble detection

\section{INTRODUCTION}

Many lab-on-a-chip technologies represent a technological backend to integrate, automate, miniaturize and parallelize complex assay protocols in the life sciences. To this end, liquid handling operations such as sample preparation, aliquoting, metering, routing and mixing are concatenated on a single (polymer) substrate. To save time and costs per assay, several reaction channels are often run simultaneously on the same substrate

However, it is well known that the reproducibility of liquid handling processes implemented by microfluidic operations tends to suffer from their extraordinary sensitivity to surface effects which frequently change in time or from batch to batch. For a successful transfer of lab-on-a-chip technologies from expert use in research labs to products in strictly regulated enduser markets such as in-vitro diagnostics (IVD), the issue of reproducibility and quality assurance is absolutely key.

In practice, the quality control of liquid handling operations often concerns the detection

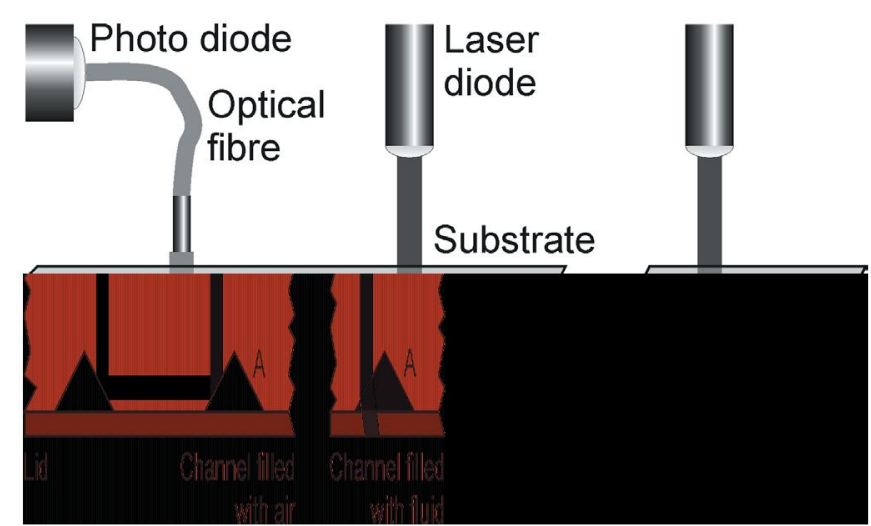

Figure 1. Schematic cross section of the detection principle. Left. A beam from a laser diode is focused onto the channel $A$. If the channel is filled with air, total internal reflection (TIR) of the beam takes place, redirecting the beam to a second, auxiliary V-groove which reflects the beam out of the substrate plane. An optical fibre guides the light to a photodiode. Right. If the channel $A$ is filled with a liquid, no TIR occurs. The beam will be refracted through the liquid and the lid, and no light (directly) reaches the photo diode.

of gas bubbles which often interfere with the functional principle as well as the verification of 
filling heights to validate (minimum) filling levels.

In this work, we present a novel detection scheme for gas bubbles and filling levels using the principle of total internal reflection (TIR). A laser beam, e.g. from a laser diode, impinges on the side walls of a channel which are sloped at $45^{\circ}$ [1]. In an unfilled channel, TIR at the air-polymer interface deflects the beam by $90^{\circ}$ into the plane of the substrate. Using TIR on auxiliary parallel channel remaining permanently empty, the beam is then deflected out of the plane, and into a simple light detector, e.g. a photo diode, adjacent to the laser. If liquid is present in the channel, the refractive index in the channel changes, and the TIR is eliminated. Instead, the beam is refracted, continuing through the substrate plane.

Such a binary, opto-fluidic light switch is robust, requiring no signal strength calibration, and even tolerates large alignment errors of more than $\pm 0.5 \mathrm{~mm}$ (for $300 \mu \mathrm{m}$ deep channels) between the laser and detector. By scanning a laser and photo diode head along a stationary channel, gas bubbles in liquid-filled channels can easily be detected.

The fabrication of the auxiliary TIR structures in optically transparent polymer substrates can readily be achieved by standard techniques such as injection molding without raising the production costs. In centrifugal microfluidic technologies, the density-dependent volume force drives the liquid entirely into the radially outer part while displacing gas bubbles towards the center of rotation. Towards elevated frequencies of rotation, the centrifugal force also prevails

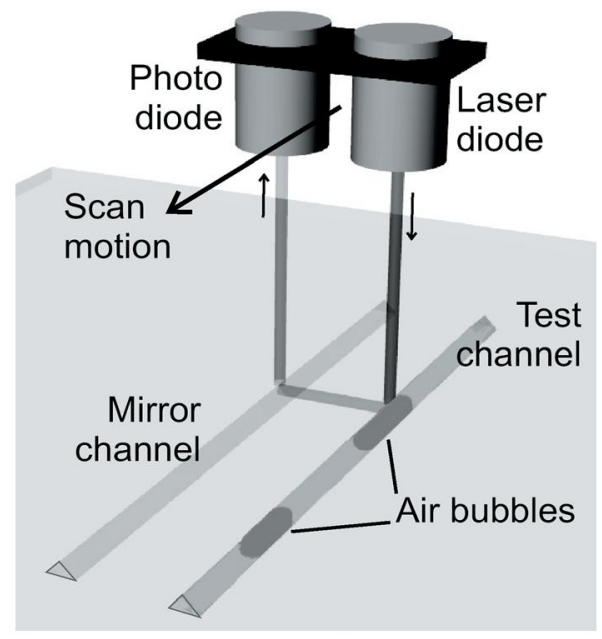

Figure 2. The laser and photo diode head scans along the channel to be investigated. The laser beam is reflected by TIR when gas bubbles are present in the channel, and is lead to the auxiliary channel, which reflects the beam into the photo diode. Where liquid is present in the channel, the laser beam is refracted through the substrate.

surface tension to flatten the meniscus. Taking into account the (known) channel geometry, the light switch thus constitutes a simple and reliable means for volume measurement.

After this introduction, the subsequent section outlines the TIR-based setup. Next, the experimental results are presented before summarizing and concluding.

\section{SETUP}

Figure 1 shows our implementation of the optofluidic switch. A beam issued by a laser diode impinges on the $45^{\circ}$-sloped side wall of the
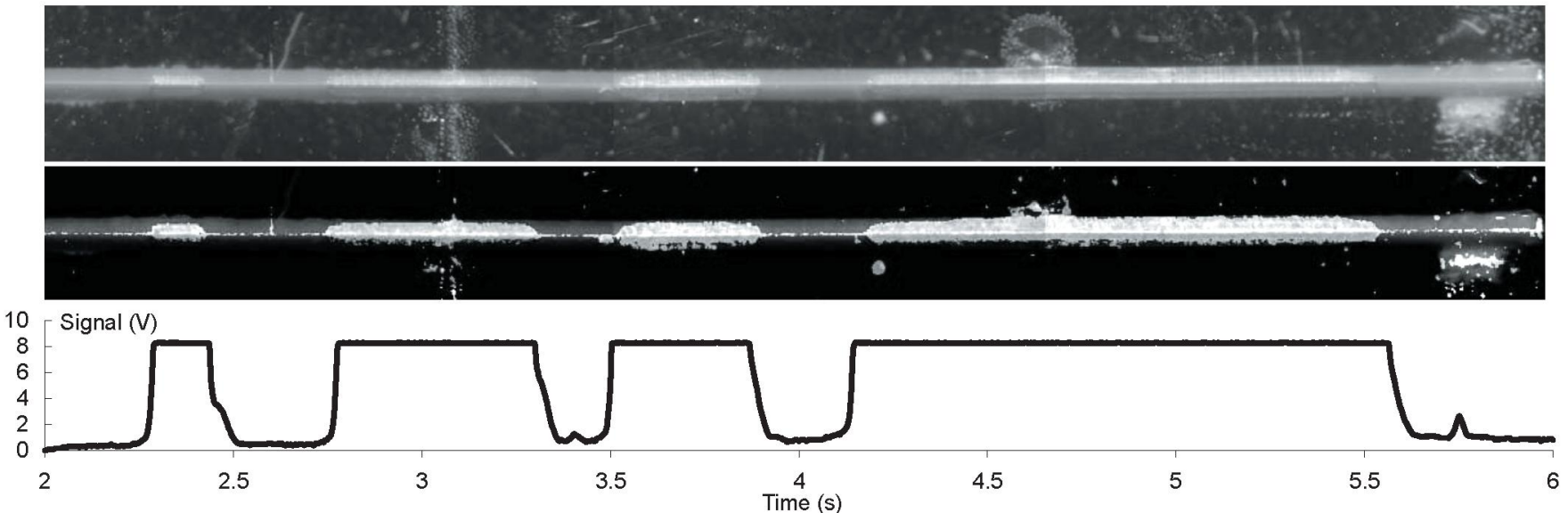

Figure 3. Bubble detection by scanning along the channel. Top: Original image of the partially filled channel. Middle: Digitally enhanced image. Air bubbles are coloured white. Bottom: The corresponding photo diode readout features an excellent agreement of $250 \mu \mathrm{m}$ with the image. 


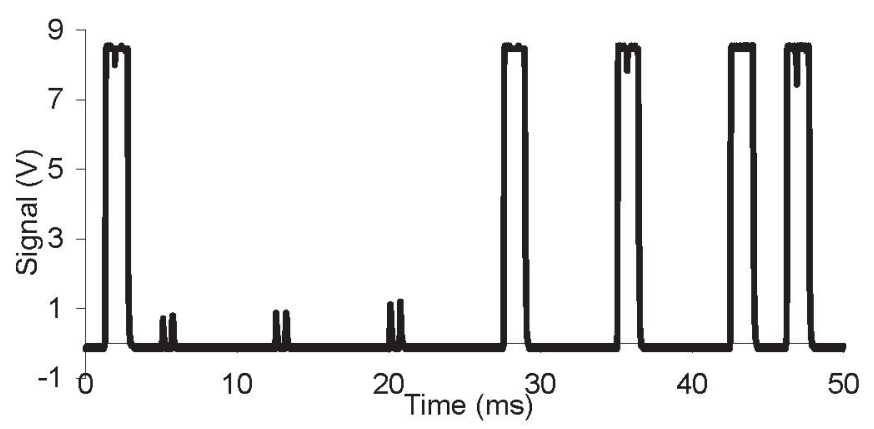

Figure 4. Photo diode readout from a rotating disk with a trigger structure and six channels separated by a $60^{\circ}$ angle (see also Fig. 4). The first three channels are filled with water, and the last three are empty, thus causing TIR. Due to the scattering on the milled channel surface, small peaks emerge when the channel is filled with water. Each channel produces two signals due to the two V-grooves adjacent to each channel; however the saturated peaks (4-6) overlap.

channel under investigation. If the channel is filled with air, the beam is deflected into the substrate plane and subsequently redirected towards an optical fiber connected a photodiode using a permanently air-filled, auxiliary V-groove. In case the channel is filled with water, no TIR takes place and the beam is refracted through the water and the lid without reaching the photodiode.

Apart from the V-grooved channels on the polymer substrate, only simple, low-cost components need to be added to the device in order to realize the opto-fluidic quality control on the device. Such components are available from CD/DVD pickup technology for a few euro only.

\section{RESULTS}

In Figures 2 and 3, the laser and the photo diode are mounted on a translational stage for recording the distribution of liquid in partially filled stationary microfluidic structures. When an empty section is interrogated by the laser, the photo diode detects a saturated signal, while a filled channel segment yields a clearly attenuated signal. By using saturated signals in $300-\mu \mathrm{m}$ deep channels, the lateral alignment of the laser and the detector in the plane of the substrate can each be varied by $\pm 0.5 \mathrm{~mm}$, until the 'filled' and 'empty' signals are indistinguishable. The vertical distance between the substrate and the fiber end, as well as the distance between the substrate and the laser, can also be shifted by several centimeters without obstructing the sensor function. Figure 3 also demonstrates excellent agreement between the channel image and the signal recorded by the opto-fluidic detector with a positional accuracy better than $250 \mu \mathrm{m}$ along the channel axis.

For use in centrifugal platforms [2], measurements on a substrate rotating at a frequency of up to $60 \mathrm{~Hz}$ have been successfully been conducted (Figs. 4 and 5). As the intrinsic centrifugal field forces the liquid phase to settle in the (radially) outer part of a dead-end vessel. Furthermore, the volume force tends to flatten the menisci of the so-compacted volumes. The optofluidic scheme thus represents a reliable volume control sensor, e.g. to indicate "sufficient sample" after priming.

Figure 4 also reveals a base noise arising from the scattering of the laser on the rough channel surface made by milling. This noise differentiates from the zero level of the detector by a factor 10 , thus serving as an internal quality control (laser on) of the error detection system.

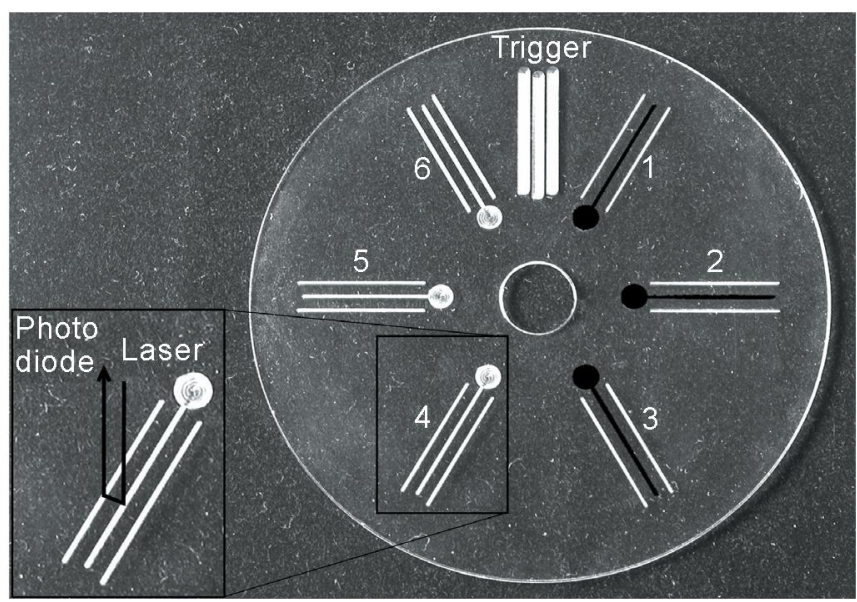

Figure 5. Compact-disc sized PMMA substrate with micro milled V-groove channels sealed by roll lamination of a lid onto the structures. Each of the six structures consists of a central channel which is filled from the reservoirs near the center of rotation, and two adjacent V-grooves. The channels 1-3 are coloured to indicate that they are water-filled. Inset: Working principle. The laser and the photo diode are placed above the rotating disk. The distance between laser and photo diode is roughly equivalent to the distance between the channels. 


\section{CONCLUSIONS AND OUTLOOK}

The opto-fluidic switch presented here provides a simple and robust means for a reliable liquid detection. Due to the uncomplicated integration of the required auxiliary channel structures in the microfabrication process of the substrate as well as the technically uncomplicated component upgrade of the device, the opto-fluidic technology can easily be incorporated in many microfluidic systems. On centrifugal platforms, the compaction of liquid volumes and the flatting of air-liquid interfaces towards high frequencies of rotation even allows to accurately meter volumes in known "dead-end" channel or chamber geometries.

After these proof-of-principle experiments, we plan on using the detector for online feedback and quality control functions in centrifugal microfluidic systems with a light source and detector resting in the lab frame.

\section{REFERENCES}

[1] M. Grumann, I. Moser, J. Steigert, L. Riegger, A. Geipel, C. Kohn, G. Urban, R. Zengerle, and J. Ducrée. "Optical Beam Guidance in Monolithic Polymer Chips for Miniaturized Colorimetric Assays". in Proc. 18th IEEE International Conference on Micro Electro Mechanical Systems, Miami, Florida, USA, January 30-February 3, 2005, pp. 108-111.

[2] J. Steigert, M. Grumann, T. Brenner, K. Mittenbühler, T. Nann, J. Rühe, I. Moser, S. Haeberle, L. Riegger, J. Riegler, W. Bessler, R. Zengerle, and J. Ducrée. "Integrated Sample Preparation, Reaction, and Detection on a Highfrequency Centrifugal Microfluidic Platform". Journal of the Association for Laboratory Automation, Vol. 10, No. 5, pp. 331-341, 2005. 